\title{
Biopsia por saturación en el diagnóstico de cáncer de próstata
}

\author{
Giménez Bachs JM, Martínez Ruiz J, Carrión López P, Martínez Sanchiz C, Pastor Navarro H, \\ Virseda Rodríguez JA.
}

Servicio de Urología. Complejo Hospitalario Universitario de Albacete.

Actas Urol Esp. 2008;32(8):787-791

\section{RESUMEN}

\section{BIOPSIA POR SATURACIÓN EN EL DIAGNÓSTICO DE CÁNCER DE PRÓSTATA}

Objetivos: Analizar los resultados obtenidos tras la realización de biopsia de próstata por saturación a una serie de pacientes con PSA elevado.

Sujetos, material y método: Durante los años 2006 y 2007 se han realizado en nuestro Servicio biopsias por saturación a 32 pacientes con PSA elevado, biopsias previas sin diagnóstico de cáncer de próstata y alta sospecha de padecerlo.

Resultados: La edad media de los pacientes fue de 65,81 años, con una media de biopsias previas de 2,41 (rango: 1 a 5). La media de PSA por el que se indicó la biopsia fue de 15,45 ng/dl (rango: 5,63 a 35,47 ng/ml). El número medio de cilindros obtenidos en las biopsias por saturación fue de 20,78 (rango: 16-26). Se diagnosticaron 13 adenocarcinomas de próstata (40,63\%), de los cuales 10 tuvieron diagnóstico previo de PIN o atipia. Se realizó prostatectomía radical a 8 pacientes (5 retropúbicas y 3 laparoscópicas), 3 casos fueron sometidos a radioterapia, 1 caso fue tratado con hormonoterapia y el restante se mantiene en seguimiento. Hubo concordancia del estadio de Gleason en 6 de los 8 pacientes intervenidos quirúrgicamente.

Conclusiones: La biopsia por saturación es un método eficaz para el diagnóstico de adenocarcinoma de próstata cuando tras varias biopsias convencionales negativas persiste una fuerte sospecha clínica de patología maligna. No debe consistir en un procedimiento de primera elección para el diagnóstico del cáncer de próstata.

Palabras clave: Cáncer de próstata. Biopsia por saturación. Diagnóstico.

\section{ABSTRACT \\ SATURATION BIOPSY IN PROSTATE CANCER DIAGNOSIS}

Aims: To analyze the results obtained after doing saturation prostate biopsy to a series of patients with high level of PSA.

Subjects, material and method: Among 2006 and 2007 saturation biopsies have been realized in our Service to 32 patients with high PSA, previous biopsies without diagnosis of prostate cancer and high suspicion of malignant disease.

Results: The mean age was 65.81 years, with an average of previous biopsies of 2.41 (range: 1 to 5). Mean of PSA was $15.45 \mathrm{ng} / \mathrm{dl}$ (range: 5.63 to $35.47 \mathrm{ng} / \mathrm{ml}$ ). The mean number of cores obtained in the saturation biopsies was of 20.78 (range: 16-26). 13 were diagnosed prostate adenocarcinomas (40.63\%), of which 10 had previous diagnosis of PIN or atipia. 8 patients underwent radical prostatectomy, 3 cases were treated with radiotherapy, 1 case was treated with hormonetherapy and the remaining one is kept in watchful waiting. Concordance of Gleason grade was in 6 of 8 patients treated surgically.

Conclusions: Saturation biopsy is an effective method for the diagnosis of prostate cancer after several negative biopsies and a strong clinical suspicion of malignant pathology. Saturation biopsy is not a first choice procedure for the diagnosis of prostate cancer.

Keywords: Prostate cancer. Saturation biopsy. Diagnosis. 
$\mathrm{E}^{1}$ diagnóstico del cáncer de próstata es uno de los más frecuentes en la consulta de un urólogo. Gracias a la aparición del PSA y posteriormente la ecografía transrectal, se ha conseguido una mayor justificación para la realización de una biopsia de próstata, sobre todo en aquéllos pacientes asintomáticos y en los que la detección precoz de cáncer de próstata va a mejorar considerablemente su pronóstico, pudiendo ofrecer al paciente un tratamiento curativo ${ }^{1}$.

Quizá uno de los problemas más preocupantes en la era del PSA es la posibilidad del sobrediagnóstico, teniendo en cuenta que se ha descrito la existencia de tumores clínicamente irrelevantes. El hecho de disponer de un arma diagnóstica específica para la detección del cáncer de próstata, como es el PSA, hace que la biopsia de próstata sea uno de los procedimientos más habituales en un servicio de Urología. Pero los límites de indicación así como la repetición de la misma quedan un poco difusos, llegando a someter a algunos pacientes a múltiples biopsias sin llegar al diagnóstico esperado ${ }^{2-4}$.

La biopsia de próstata por saturación es el procedimiento que persigue la detección del cáncer de próstata a costa de obtener un número de muestras de tejido prostático muy superior al habitual. Con el presente trabajo pretendemos analizar la utilidad de este procedimiento así como orientar el momento en que debe indicarse.

\section{SUJETOS, MATERIAL Y MÉTODO}

Durante los años 2006 y 2007 se han realizado en nuestro servicio 32 biopsias por saturación a pacientes con PSA elevado y con al menos una biopsia previa sin diagnóstico definitivo de cáncer de próstata.

A los pacientes a los que se les realizó la biopsia por saturación se les administró antibioticoterapia profiláctica, así como enemas de limpieza rectal. Todas las biopsias por saturación se realizaron en el quirófano bajo sedación o raquianestesia, y de manera ecodirigida se obtuvieron las muestras de la próstata, a criterio del urólogo que realizaba el procedimiento y dependiendo del tamaño de la próstata. Los pacientes permanecían con sonda vesical durante 24 horas tras la biopsia.

Se ha realizado un estudio observacional retrospectivo a partir de las historias clínicas de los pacientes, obteniendo los datos sociodemográficos de los mismos así como datos en relación a su patología prostática: número de biopsias de próstata previas, cifras de PSA, resultado anatomopatológico de las biopsias y tratamiento al que se sometieron los pacientes diagnosticados de cáncer de próstata. Los datos obtenidos fueron analizados mediante el programa estadístico SPSS 15.0.

\section{RESULTADOS}

La edad media de los pacientes fue de 65,81 años (DE: 5,74; IC95\%:63,74-67,88 años). La media de biopsias realizadas previas a la de saturación fue de 2,41 (Rango: 1 a 5). La media de PSA por el que se indicó la biopsia por saturación fue de 15,45 ng/dl (DE: 8,76; IC95\%: 12,29-18,61 ng/dl; Rango: $5,63$ a $35,47 \mathrm{ng} / \mathrm{ml})$. Se obtuvieron una media de 20,78 (Rango: 16-26) cilindros de tejido prostático en dichas biopsias.

Los resultados de la histopatología en las biopsias convencionales previas se muestran en la Figura 1.

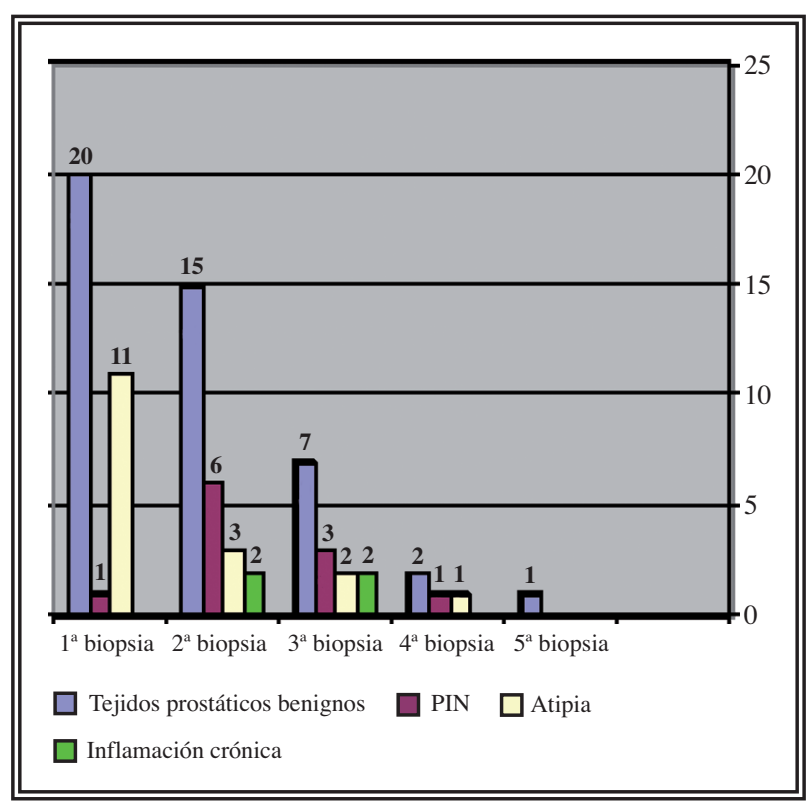

FIGURA 1. Anatomía Patológica de las biopsias realiza das antes de la indicación de la biopsia por saturación.

Se diagnosticaron 13 adenocarcinomas de próstata, lo que supone un 40,63\% del total. Se diagnosticó cáncer de próstata en el 38,46\%, 46,15\% y $15,38 \%$ de los pacientes que llevaban 2, 3 y 4 biopsias previas negativas respectivamente, mientras que en ningún paciente con una sola biopsia previa ni en el único con 5 biopsias anteriores se llegó al diagnóstico de malignidad. Los diagnósticos histopa- 
tológicos obtenidos en el total de las biopsias por saturación se muestran en la Figura 2. De los 13 pacientes diagnosticados de adenocarcinoma, 6 tuvieron en alguna de las biopsias previas el diagnóstico de atipia sospechosa de malignidad y 4 el de PIN.

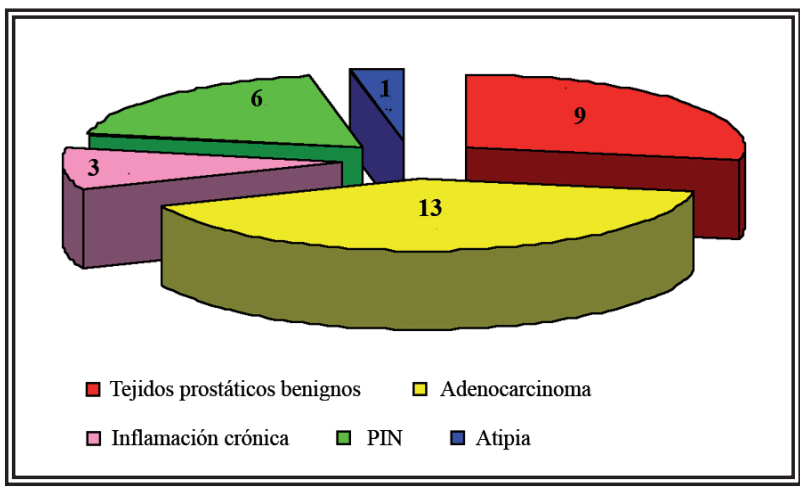

FIGURA 2. Anatomía patológica de las biopsias por saturación.

En la Tabla 1 se muestran los distintos diagnósticos dependiendo del número de cilindros obtenidos. No hubo relación estadísticamente significativa entre ambas variables, quizá por el reducido número de la muestra. No hubo complicaciones en ninguno de los pacientes.

De los pacientes diagnosticados de adenocarcinoma de próstata se realizó prostatectomía radical a 8 pacientes (5 retropúbicas y 3 laparoscópicas), 3 casos fueron sometidos a radioterapia, 1 caso fue tratado con hormonoterapia y el restante se mantiene en seguimiento, con determinaciones de PSA periódicas. Hubo concordancia del estadio de Gleason en 6 de los 8 pacientes intervenidos quirúrgicamente, los dos pacientes restantes fueron supraestadiados, ya que uno reveló Gleason 6 definitivo (en biopsia previa fue 7 ) y el otro Gleason 5 (en biopsia previa fue 6).

\section{DISCUSIÓN}

El diagnóstico de cáncer de próstata sólo puede ser establecido mediante el estudio histológico del tejido prostático. La aparición de la ecografía transrectal y la toma de muestras ecodirigidas ha aumentado considerablemente el rendimiento diagnóstico de la biopsia de próstata ${ }^{1}$. En un principio se estableció la biopsia por sextantes ${ }^{5}$ y ha sido durante mucho tiempo el método utilizado a la hora de realizar la biopsia. Posteriormente, se han ido describiendo distintas tácticas de biopsia, con el fin de aumentar la capacidad diagnóstica, de manera que hoy en día existen múltiples opciones para la toma de muestras, intentando "mapear" de manera más exhaustiva la próstata, teniendo en cuenta los lugares más frecuentes de asiento del cáncer de próstata. En cualquier caso, prácticamente todas estas nuevas variantes de la biopsia por sextantes han aumentado el número de muestras, estando aconsejado, hoy en día, la toma de 10 a 12 cilindros de tejido $^{6}$.

A pesar de aumentar el número de cilindros de tejido obtenidos en la biopsia prostática, existe un porcentaje no desdeñable de pacientes a los que no conseguimos detectarles el cáncer, aun cuando la sospecha de padecer dicha enfermedad es alta, lo que hace que repitamos la biopsia múltiples veces hasta conseguir el diagnóstico o hasta desistir (ya el paciente, ya el urólogo). La rentabilidad diagnóstica de la biopsia de próstata es inversamente proporcional al número de repeticiones de la misma, por lo que se aconseja seleccionar mucho a los pacientes

Tabla 1. Diagnóstico histológico en relación con el número de cilindros obtenidos.

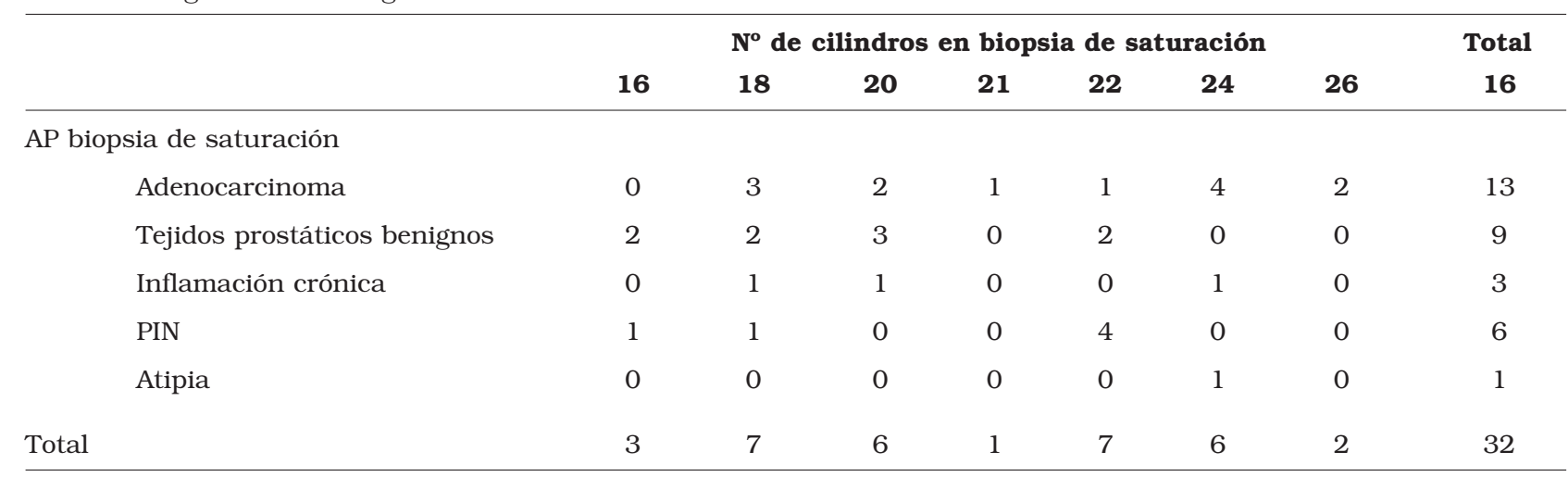


que queremos realizarles la tercera o cuarta biopsia, ya que además, los cánceres detectados en estos pacientes suelen ser de menor grado ${ }^{7}$. Es cuando, tras varias biopsias negativas y persistiendo una alta sospecha de cáncer, recurrimos a la denominada biopsia por saturación, aumentando considerablemente el número de cilindros obtenidos (llegando, en ocasiones, a más de 40), mejorando la capacidad diagnóstica de la biopsia ${ }^{8}$.

Pero la biopsia de saturación no debe realizarse como primera opción, es decir, aunque se ha demostrado que mejora la detección de cáncer de próstata después de una o varias biopsias previas negativas, no parece ofrecer beneficio como técnica de biopsia inicial $^{9}$.

Por tanto la biopsia por saturación es un método eficaz cuando se realiza en determinados pacientes, pero nos enfrentamos a la posibilidad de diagnosticar tumores denominados clínicamente insignificantes, es decir, aquéllos con un volumen menor a 0,2 cm3, grado de Gleason 4 ó 5, y ausencia de extensión extraprostática o afectación ganglionar ${ }^{4,10}$. Con la biopsia por sextantes, parece ser que la probabilidad de detectar cáncer de estas características es menor, ya que muchos de los tumores a priori con estadios más bajos, en la pieza de prostatectomía se demostró un estadio real más alto, lo que se debe tener en cuenta a la hora de plantear un tratamiento ${ }^{2}$.

Un tema controvertido es la significación clínica de la atipia glandular y la neoplasia prostática intraepitelial (PIN). Dichas entidades no tienen un tratamiento establecido, aunque algunos autores defienden el tratamiento del PIN con deprivación androgénica para reducir su progresión ${ }^{11}$. En cualquier caso, lo que parece claro es la progresión de estas lesiones hacia el cáncer, de manera que pacientes diagnosticados de PIN pueden desarrollar un carcinoma prostático hasta en un $40 \%$ de los casos en posteriores biopsias $^{12}$, alcanzando hasta el $80 \%$ si se trata de un PIN de alto grado multifocal ${ }^{13}$, con más probabilidad de detectarlas si se realizan biopsias extendidas o por saturación. No obstante, parece ser que la patogenicidad de estas entidades es distinta, de manera que los pacientes con PIN tienen cierta probabilidad de tener cáncer en otra localización prostática, no detectado por la biopsia, mientras que es en la atipia cuando biopsias posteriores en el mismo lugar detectan un cáncer por progre- sión ${ }^{6}$. En nuestra serie, podemos observar como el diagnóstico de PIN y/o atipia decrece conforme se realizan más biopsias, puesto que al repetir biopsias se detectan cáncer de próstata en pacientes con estos diagnósticos previos (10 de 13, lo que supone un $76,9 \%$ ).

Otro punto de discusión sería el número de cilindros a obtener y la localización de los mismos. Parece claro que al menos 20 cilindros deben ser obtenidos en este tipo de biopsia, aunque el tamaño prostático es importante a la hora de decidirlo, puesto que serán necesarias más muestras cuanto mayor tamaño tiene la próstata ${ }^{14}$. También es importante la localización de la próstata donde hay que tomar más muestras por representar los lugares más frecuentes de detección del cáncer. Obtener muestras más laterales de la zona periférica y del ápex aumenta el rendimiento diagnóstico, mientras que, aunque las biopsias por saturación incluyen la toma de cilindros en la zona de transición, esta región es asiento poco frecuente de cáncer y en muchas ocasiones de los denominados clínicamente insignificantes ${ }^{10,15,16}$. No obstante, en la primera biopsia de próstata, la toma de cilindros en la zona parasagital puede ser importante para la detección del cáncer en comparación con los pacientes a los que se les detecta en biopsias de repetición ${ }^{17}$.

Las complicaciones que aparecen tras una biopsia de próstata por saturación son generalmente las derivadas del sangrado (uretral o rectal), las infecciones o la retención aguda de orina. Una hemorragia que precise hospitalización es muy infrecuente, y los sangrados suelen ser autolimitados, teniendo en cuenta que la uretrorragia es rara si se deja una sonda vesical durante unas horas tras realizar la biopsia El mayor número de cilindros obtenidos aumenta la probabilidad de retención aguda de orina, por lo que se suele dejar sonda vesical para evitarlo $^{16}$. La profilaxis antibiótica disminuye considerablemente las complicaciones infecciosas, y el continuar con antibiótico durante 3 días tras la biopsia no parece ofrecer beneficios en este aspecto ${ }^{18}$. El dolor es otra de las posibles complicaciones, aunque en esta técnica, el hecho de hacerse con anestesia regional disminuye los problemas derivados del dolor, si bien se ha descrito la posibilidad de realizar biopsias por saturación con bloqueo periprostático con anestesia local ${ }^{19}$. En nuestra serie, no ha habido complicaciones destacables, lo que 
reafirma que este procedimiento es seguro en este aspecto, si bien, el limitado número de pacientes que presentamos no hace posible la generalización.

Por tanto, la biopsia por saturación es una técnica que debe estar indicada en aquellos pacientes que tras una o varias biopsias previas negativas, persiste la sospecha clínica de cáncer de próstata, bien por PSA elevado, tacto rectal sospechoso, o diagnóstico anterior de PIN y/o atipia ${ }^{8}$. Se consigue un aumento en la detección del cáncer con respecto a otros esquemas y con cierta seguridad en relación a la morbilidad ${ }^{3,6,8,10}$, si bien existen autores que consideran suficiente la toma de un número extendido de muestras (12 a 18 cilindros) en la zona periférica en aquéllos pacientes que no hemos conseguido demostrar la presencia de un cáncer a pesar de una fuerte sospecha ${ }^{21}$.

En conclusión, la biopsia de próstata por saturación constituye una modalidad más para el diagnóstico de cáncer de próstata, que debe ser indicada en un determinado tipo de pacientes y no como rutina. Si tras la realización de una biopsia de este tipo, el resultado es negativo debemos plantearnos el seguimiento con métodos menos cruentos, incluso, en casos concretos desestimar la existencia de patología maligna prostática.

\section{REFERENCIAS}

1. Ramírez Backhaus M, Trassierra Villa M, Arlandis Guzmán S, Delgado Oliva F, Boronat Tormo F, Jiménez Cruz JF. Estrategias para la biopsia de próstata. Revisión de la literatura. Actas Urol Esp. 2007;31(10):1089-1099.

2. Luján Galán M, García Tello A, Pascual Mateo C, Llanes González L, García Medeiro JM, Angulo Cuesta J et al. Prevalencia y significado clínico de los cánceres de próstata focales e incidentales. Actas Urol Esp. 2007;31(8):819-24.

3. Scattoni V, Zlotta A, Montironi R, Schulman C, Rigatti P, Montorsi F. Extended and saturation prostatic biopsy in the diagnosis and characterisation of prostate cancer: a critical analysis of the literature. Eur Urol. 2007;52(5):1309-22.

4. Epstein JI, Sanderson H, Carter HB, Scharfstein DO. Utility of saturation biopsy to predict insignificant cancer at radical prostatectomy. Urology. 2005;66(2):356-360.

5. Hodge KK, McNeal JE, Terris MK, Stamey TA. Random systematic versus directed ultrasound guided transrectal core biopsies of the prostate. J Urol. 1989;142(1):71-75.

6. Presti JC Jr. Prostate biopsy strategies. Nat Clin Pract Urol. 2007 4(9):505-511.
7. Djavan B, Ravery V, Zlotta A, Dobronski P, Dobrovits M, Fakhari $\mathrm{M}$ et al. Prospective evaluation of prostate cancer detected on biopsies 1, 2, 3 and 4: When should we stop?. J Urol. 2001;166 (5): 1679-1683.

8. Stewart CS, Leibovich BC, Weaver AL, Lieber MM. Prostate cancer diagnosis using a saturation needle biopsy technique after previous negative sextant biopsies. J Urol. 2001;166(1):86-91.

9. Jones JS, Patel A, Schoenfield L, Rabets JC, Zippe CD, MagiGalluzzi C. Saturation technique does not improve cancer detection as an initial prostate biopsy strategy. J Urol. 2006;175:485-488.

10. Chrouser KL, Lieber MM. Extended and saturation needle biopsy for the diagnosis of prostate cancer. Curr Urol Rep. 2004;5(3):226-230

11. Steiner MS. High grade prostatic intraepithelial neoplasia is a disease. Curr Urol Rep. 2001;2(3):195-198.

12. Langer JE, Rovner ES, Coleman BG, Yin D, Arger PH, Malkowicz SB et al. Strategy for repeat biopsy of patients with prostatic intraepithelial neoplasia detected by prostate needle biopsy. J Urol. 1996;155(1):228-231.

13. Schoenfield L, Jones JS, Zippe CD, Reuther AM, Klein E, Zhou $\mathrm{M}$, et al. The incidence of high-grade prostatic intraepithelial neoplasia and atypical glands suspicious for carcinoma on first-time saturation needle biopsy, and the subsequent risk of cancer. BJU Int. 2007;99(4):770-774.

14. Merrick GS, Gutman S, Andreini H, Taubenslag W, Lindert DL, Curtis $\mathrm{R}$ et al. Prostate cancer distribution in patients diagnosed by transperineal template-guided saturation biopsy. Eur Urol. 2007;52(3):715-723.

15. Jones JS. Saturation biopsy for detecting and characterizing prostate cancer. BJU Int. 2007;99(6):1340-1344.

16. Raja J, Ramachandran N, Munneke G, Patel U. Current status of transrectal ultrasound-guided prostate biopsy in the diagnosis of prostate cancer. Clin Radiol. 2006;61(12):142-153.

17. Patel AR, Jones JS, Zhou M, Schoenfield L, Magi-Galluzzi C. Parasagittal biopsies are more important as part of an initial biopsy strategy than as part of a repeat biopsy strategy: observations from a unique population. Prostate Cancer Prostatic Dis. 2007;10(4):352-355.

18. Aron M, Rajeev TP, Gupta NP. Antibiotic prophylaxis for transrectal leedle biopsy of the prostate: a randomized controlled study. BJU Int. 2000;85(6):682-685.

19. Jones JS, Oder M, Zippe CD. Saturation prostate biopsy with periprostatic block can be performed in office. J Urol. 2002;168 (5):2108-2110.

20. Moon K, Theodorescu D. Does saturation biopsy improve prostate cancer detection?. Nat Clin Pract Urol. 2006;3(9):468-469.

21. Fleshner N, Klotz L. Role of "saturation biopsy" in the detection of prostate cancer among difficult diagnostic cases. Urology. 2002;60(1):93-97.

Correspondencia autor: Dr. J.M. Giménez Bachs

Servicio de Urología. Complejo Hospitalario Universitario de

Albacete. Hermanos Falcó, 37 - 02006 Albacete

Tel.: 967597100

E-mail autor: gbjosem@sescam.jccm.es

Información artículo: Original - Cáncer de próstata

Trabajo recibido: enero 2008

Trabajo aceptado: abril 2008 